\title{
Involvement of Gastrin-Releasing Peptide (GRP) and Gastrin-Releasing Peptide Receptor (GRPR) in the mouse model of allergic rhinitis
}

\author{
Hidenori Yokoi, Yuma Matsumoto, Koichiro Saito \\ Department of Otorhinolaryngology, Kyorin University School of Medicine, Tokyo, Japan
}

\begin{abstract}
Introduction: Gastrin-releasing peptide (GRP), a neuropeptide, and its receptor (GRPR) are reported to be involved in the inflammation and transmission of the itch sensation in the dorsal horn of the spinal cord. They have also been implicated to play a role in allergic diseases such as asthma and atopic dermatitis. However, their role in allergic rhinitis (AR) remains unknown.
\end{abstract}

The present study aimed to elucidate the role of GRP in the pathological process of AR.

Method: BALB/c female mice were sensitized with ovalbumin (OVA) in 4 different protocols: control mice were only given phosphate buffered saline (PBS), systemic sensitization only mice were given OVA systemically followed by nasal administration of PBS, mild AR mice were given OVA systemically followed by nasal administration of OVA for 1 week then PBS for another 1 week, full-blown AR mice were given OVA systemically flowed by 2 weeks of intranasal
OVA. GRP and GRPR expression levels were examined by western blotting and immunostaining at the end of each treatment. Finally, GRPR antagonist (RC-3095) was administered to full-brown AR mice.

Results: GRP was expressed in the nasal mucosal epithelium and the interstitial tissue surrounding the nasal gland and GRPR was expressed in the nasal mucosal epithelium and submucosal gland. Both GRP and GRPR expression were increased in both mild and full-blown AR mice. Moreover, intranasal administration of RC-3095 alleviated sneezing in full-blown AR mice.

Conclusion: GRP may act as an aggravating factor in AR and its inhibition may be a novel strategy to control AR.

E-mail: h-yokoi@ks.kyorin-u.ac.jp 